\title{
Model for analyzing the effects of processing on recording material in thick holograms
}

\author{
A. Beléndez, I. Pascual, and A. Fimia \\ Laboratorio de Optica, Departamento Interuniversitario de Optica, \\ Universidad de Alicante, Apartado 99, Alicante E 03080, Spain
}

\begin{abstract}
Received September 26, 1991; revised manuscript received February 3, 1992; accepted February 10, 1992
A new model to describe the influences of chemical processing on thick holograms is presented. The model is based on three hypotheses about the behavior of holographic recording material during processing. Thickness variations and shear-type effects that are due to processing are taken into account in this model, and a new parameter, the effective thickness, is introduced. It is possible to use this thickness to express the relation that exists between the components of the grating vector before and after processing. Experimental results obtained with transmission gratings recorded in a bleached silver halide emulsion are presented and discussed.
\end{abstract}

\section{INTRODUCTION}

With recent advances in holographic technology, many types of holographic optical elements (HOE's) are being used in different optical systems. Usually these elements are recorded as thick phase holograms, and two important questions associated with the making of these HOE's exist. One question concerns the design and the analysis of the HOE, and the second concerns the material used to record the HOE. In this paper we consider an important aspect of the second question: variations in the average refractive index and deformations of the recording material introduced by its processing. These changes produce a reordering of the internal structure of the interference fringes; thus we can see that the processed and the recording elements are different. As a result of these variations the reconstruction geometry corresponding to maximum efficiency has to be changed with respect to the exposure geometry, which evidently gives rise to the appearance of aberrations in holographic lenses or mirrors during the reconstruction stage.

To explain the thickness change that is due to the chemical processing of a holographic recording material, there is a model that has been commonly accepted for more than 20 years. We are referring to the model proposed by Vilkomerson and Bostwick. ${ }^{1}$ They state that, if an emulsion is bound to a rigid glass substrate, shrinkage (or swelling) is manifested as a change in emulsion thickness, and recorded fringe planes therefore rotate. Moreover, this change in the net volume of the emulsion does not change the surface pattern. Using this model, Mikhailov obtained mathematical expressions ${ }^{2}$ for the reconstruction geometry that complies with Bragg's law. This model has been tested through experimentation, and good results ${ }^{1}$ have been achieved, especially in reflection holography. ${ }^{3}$ However, there are some situations in which the theoretical results obtained by following Vilkomerson et al. are different from the expected experimental results. Syms and Solymar" analyzed holographic lenses that have small-slant fringes (recording geometry close to a symmetrical setup) and that were recorded in a bleached silver halide emulsion, obtaining levels of shrinkage of $\sim 28 \%$ with the previous shrinkage model. ${ }^{1}$ This level of shrinkage implies that $T=0.72(T=$ final thickness/initial thickness), but this value is not possible experimentally with the processing that was used. ${ }^{4}$ No explanation could be offered for this level of shrinkage, and Syms and Solymar ${ }^{4}$ commented that details about the shrinkage mechanism remain unclear. We believe that the details of the deformation mechanism of the recording material must be explained. In previous experiments ${ }^{5}$ we obtained $T \sim 0.7$ for transmission gratings with smallslant fringes when using a rehalogenating bleach. Physically this value is not possible because the thickness change introduced with this bleaching is small. ${ }^{3}$ It is important to take into account that the maximum level of shrinkage of the photographic emulsion layers is $\sim 15 \%$ $(T \sim 0.85)$ of its original thickness. ${ }^{1,6}$ Other authors ${ }^{7}$ have obtained $T \sim 0.7$ for photographic emulsions. Therefore the model proposed by Vilkomerson et al. is not correct, and it is necessary to develop a model that is capable of justifying the anomalous values for shrinkage.

To study the influence of processing on a thick holographic grating, we have developed a geometric model [the effective holographic grating model (EHGM)] in which we associate a recorded and a processed grating and an effective diffraction grating. The effective grating is determined by the grating vector after processing and has an effective thickness. This thickness determines the direction of the reconstruction beam when Bragg's law is complied with. The model has been experimentally analyzed by using diffraction gratings made with different recording geometries and exposures with processings typical of bleached emulsions and by studying the variations in the replay angle corresponding to maximum efficiency. These angle variations have been quantitatively related to the effective thicknesses of the model. We used silver halide emulsions because these are an important medium for testing holographic concepts. ${ }^{8}$ This is due to the relatively high sensitivity and ease of processing of this material, improved processing chemistries, and the repeatability of the results. 
In Section 2 the effects of chemical processing on recording material are briefly described. The more-important consequences of these effects are discussed, and the hypotheses of our model are presented. In Section 3 the theoretical development of the EHGM is presented. The relationships between the points of the holographic grating and between the grating vectors before and after processing are obtained. In Section 4 we introduce the effective thickness, and we relate this new parameter to the reconstruction geometry that complies with Bragg's law for a holographic grating. Some theoretical results are presented in Section 5, while in Section 6 we analyze the experimental results obtained with transmission gratings recorded in a bleached photographic emulsion. The results are briefly discussed. Finally, our conclusions are presented in Section 7.

\section{DESCRIPTION OF THE EFFECT OF PROCESSING ON THE RECORDING MATERIAL}

\section{A. Consequences of the Photochemical Processing for Holographic Optical Elements}

HOE's are usually made in thick phase materials because only thick phase holograms can produce efficiencies of nearly $100 \% .^{9}$ In the chemical processing of these materials we use liquid solutions. As a consequence, there is a change in the average refractive index, a deformation of the recording material, and a nonuniformity of the interference fringes through the material thickness. ${ }^{10}$

The change in the average refractive index produces a variation in the replay angle for maximum efficiency. This effect is small, ${ }^{4}$ and it can be compensated for by rotating the grating, but total compensation for holographic lenses is not possible.

The deformation phenomenon is characteristic of gelatin-based materials with liquid solution processing because the gelatin is elastic in behavior, and stresses on the volume of the material appear during processing. If we record a holographic grating, the periodic pattern recording will modify the elastic behavior of the medium. Likewise, changes in the gelatin will modify the recorded pattern. To compensate for these elastic forces, the Bragg planes rotate, and the grating vectors before and after processing are different.

For phase holograms the uniformity of the grating is due to a constant modulation in the refractive index throughout the hologram. However, this situation is usually difficult to obtain, because the light beams are progressively attenuated as they are propagated through the recording medium. ${ }^{10}$ Therefore nonuniformity of the interference fringes through the thickness is introduced when the hologram is recorded. After processing, this nonuniformity can produce a bending of the interference fringes. ${ }^{11}$ This curvature or bending of Bragg planes causes sidelobe asymmetries around the Bragg angle in the angular sensitivity curves of a thick holographic grating. ${ }^{11}$ In our model we do not consider the possible bending of interference fringes.

\section{B. Hypotheses of the Model}

We are studying the recording medium in the form of a thin layer of material on a glass backing, and, to quantify the refractive-index variation and the deformation of the holographic grating that is due to processing, we introduce a model based on three hypotheses:

Hypothesis 0 . There is a change in the average refractive index of the material that is due to processing. $n_{R}$ and $n_{C}$ are the indices of the medium before and after processing, respectively.

Hypothesis 1. There is a homogeneous deformation of the recording material: The Bragg planes before and after processing are planes.

Hypothesis 2. Owing to processing, the positions of the points in the medium in contact with the glass backing do not change. If these points were changed, the grating would be destroyed.

\section{Parameters of a Holographic Grating}

Let us consider the Bragg planes in a grating recorded in the air by two plane waves, as shown in Fig. 1 . $\mathbf{K}$ is oriented perpendicular to the Bragg planes and is the grating vector. Its modulus is $|\mathbf{K}|=K=2 \pi / \Lambda$, where $\Lambda$ is the grating spacing. We write the Cartesian components of $\mathbf{K}$ as $K_{x}=-K \cos \phi$ and $K_{z}=K \sin \phi$, where $\phi$ is the slant angle. We introduce the unit vectors $\hat{i}, \hat{j}$, and $\hat{k}$, and the vectors $\mathbf{d}_{x}=d_{x} \hat{i}$ and $\mathbf{d}_{z}=d_{z} \hat{k}$ have modulus $d_{x}$ and $d_{z}$, respectively, where $d_{x}$ and $d_{z}$ are the distances between the Bragg planes in the $X$ and the $Z$ directions, respectively. $t_{R}$ is the initial thickness of the medium. The expression for the grating vector is ${ }^{9} \mathbf{K}=\hat{k}_{R}{ }^{\prime}-\hat{k}_{O}$, where $\hat{k}_{R}{ }^{\prime}$ and $\hat{k}_{O}{ }^{\prime}$ are the propagation vectors of the reference $(R)$ and the object $(O)$ beams in the medium of index $n_{R}$, respectively. $\mathbf{K}$ can be written as

$$
\mathbf{K}=\frac{2 \pi n_{R}}{\lambda_{R}}\left[\begin{array}{c}
\sin \alpha_{R}{ }^{\prime}-\sin \alpha_{O^{\prime}} \\
0 \\
\cos \alpha_{R}{ }^{\prime}-\cos \alpha_{O^{\prime}}
\end{array}\right] .
$$

It is possible to write this vector as

$$
\mathbf{K}=2 \pi\left(\frac{1}{d_{x}} \hat{i}+\frac{1}{d_{z}} \hat{k}\right) .
$$

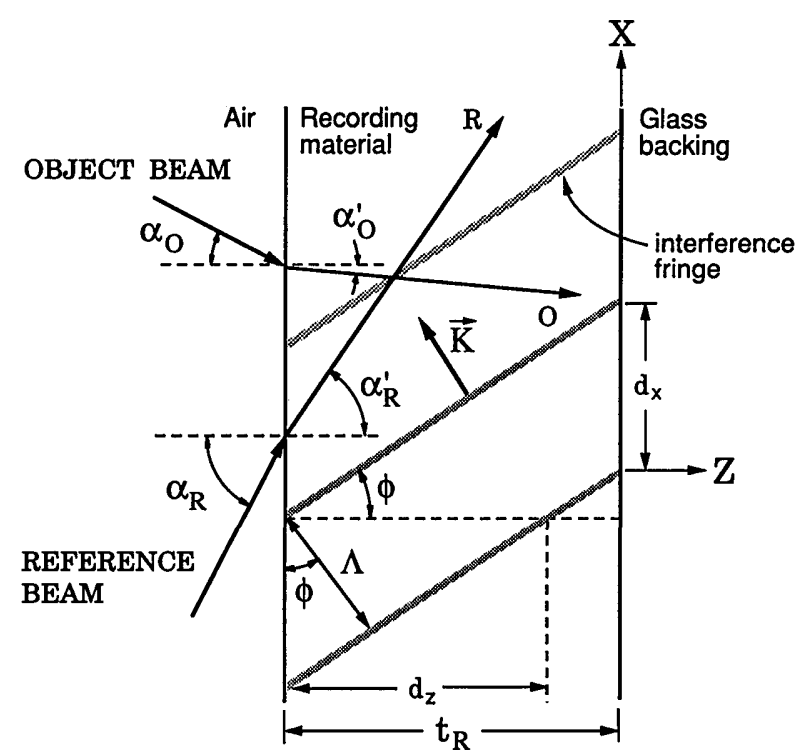

Fig. 1. Recording parameters of a thick transmission grating. 


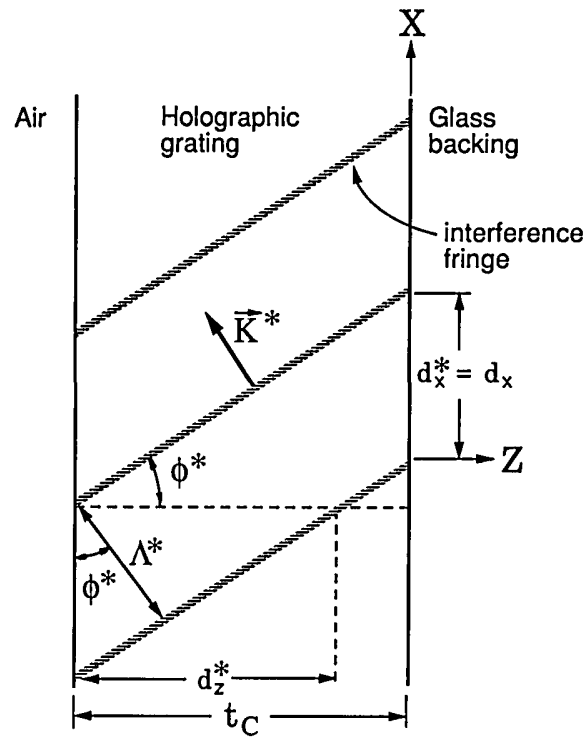

Fig. 2. Holographic grating after processing.

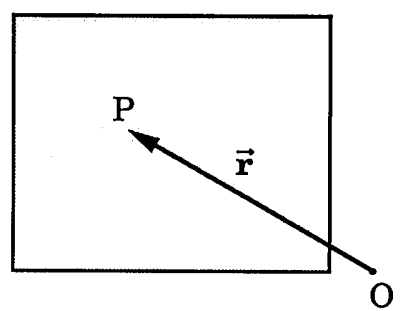

(a)

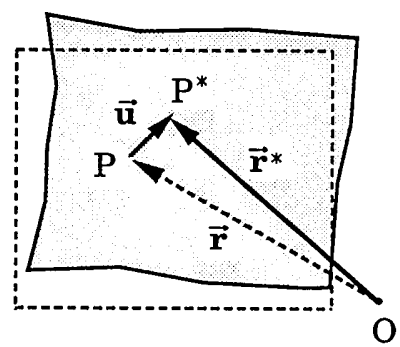

(b)
Fig. 3. Situation of a point (a) before and (b) after deformation of the medium owing to processing.

We consider that the Bragg planes change size but do not change shape (hypothesis 1), so that they end up being planes that are parallel and equidistant. Likewise, a grating after processing (Fig. 2) is different from the recorded grating. We use an asterisk to denote the index of the parameters of the grating after processing: $\mathbf{K}^{*}, \phi^{*}$, $\Lambda^{*}$, and $d_{x}{ }^{*}$ and $d_{z}{ }^{*}$. Moreover, $t_{C}$ and $n_{C}$ are the new thickness and index, respectively. According to hypothe$\operatorname{sis} 2, d_{x}^{*}=d_{x}$.

\section{THEORETICAL DEVELOPMENT OF THE MODEL}

The problem is to obtain the more general relationship between the components of vectors $\mathbf{K}$ and $\mathbf{K}^{*}$ when the above hypotheses are satisfied. Let us consider a part of the recording material before processing [Fig. 3(a)], corresponding to a holographic grating. A point $\mathrm{P}$ of this medium is determined by a vector $\mathbf{r}$. During processing of the medium and owing to the chemical reactions that take place, the material no longer maintains its original state of equilibrium. As a result, internal stresses appear that tend to push the medium toward a state that is new yet still balanced. To compensate for these elastic forces, the Bragg planes rotate, and the thickness of the medium varies. $\mathrm{P}$ occupies a new position that we call $\mathrm{P}^{*}$, identified by using vector $\mathbf{r}^{*}$ [Fig. 3(b)]. 'I'he displacement of a point of the medium can be determined by using the displacement vector $\mathbf{u}=\mathbf{r}^{*}-\mathbf{r}=\Delta x \hat{i}+\Delta y \hat{j}+\Delta z \hat{k}$. The simplest geometric transformation that satisfies hypothesis 1 of the model is a linear transformation that can be written as

$$
\left[\begin{array}{l}
x^{*} \\
y^{*} \\
z^{*}
\end{array}\right]=\left[\begin{array}{l}
a_{10} \\
a_{20} \\
a_{30}
\end{array}\right]+\left[\begin{array}{lll}
a_{11} & a_{12} & a_{13} \\
a_{21} & a_{22} & a_{23} \\
a_{31} & a_{32} & a_{33}
\end{array}\right]\left[\begin{array}{l}
x \\
y \\
z
\end{array}\right],
$$

with $\alpha_{i j}$ being constants.

Hypothesis 2 shows that $d_{x}$ and $d_{y}$ remain constant after processing. If these distances do not vary, the processing of the medium does not change either the surface pattern of the interference fringes or the direction of the diffracted beam. To express this condition we take into account the fact that the points that pertain to the medium-support contact plane (plane $z=0$ ) are written as $\mathrm{P}(x, y, 0)$. After processing, the transformed point $\mathrm{P}^{*}$ should have the same coordinates as $\mathrm{P}$, and thus $x^{*}=x, y^{*}=y$, and $z^{*}=0$. Taking this into account, we can rewrite Eq. (3) as

$$
\left[\begin{array}{l}
x^{*} \\
y^{*} \\
z^{*}
\end{array}\right]=\left[\begin{array}{lll}
1 & 0 & a \\
0 & 1 & b \\
0 & 0 & c
\end{array}\right]\left[\begin{array}{l}
x \\
y \\
z
\end{array}\right]
$$

where $a, b$, and $c$ are constants whose physical representations are shown below. By applying Eq. (3) to vectors $\mathbf{d}_{x}$, $\mathbf{d}_{y}$, and $\mathbf{d}_{z}$, we obtain

$$
\left[\begin{array}{lll}
\mathbf{d}_{x}^{*} & \mathbf{d}_{y}{ }^{*} & \mathbf{d}_{z}^{*}
\end{array}\right]=\left[\begin{array}{lll}
\mathbf{d}_{x} & \mathbf{d}_{y} & \mathbf{d}_{z}
\end{array}\right]\left[\begin{array}{lll}
1 & 0 & a \\
0 & 1 & b \\
0 & 0 & c
\end{array}\right] .
$$

Additionally, $\mathbf{K}$ is a reciprocal grating vector and thus belongs to the dual space of the vectorial space of the medium's vectors. Therefore $\mathbf{K}$ and $\mathbf{K}^{*}$ are related as follows:

$$
\left[\begin{array}{lll}
K_{x}^{*} & K_{y}{ }^{*} & K_{z}{ }^{*}
\end{array}\right]=\left[\begin{array}{lll}
K_{x} & K_{y} & K_{z}
\end{array}\right]\left[\begin{array}{ccc}
1 & 0 & -(a / c) \\
0 & 1 & -(b / c) \\
0 & 0 & 1 / c
\end{array}\right] .
$$

This relation indicates that the component $Z$ of the grating vector after processing is a function of the components $X$, $Y$, and $Z$ of the grating vector before processing, while the $X$ and the $Y$ components of $\mathbf{K}$ do not change.

Equations (4) and (5) relate the coordinates of the points of the medium and vectors $\mathbf{d}_{x}, \mathbf{d}_{y}$, and $\mathbf{d}_{z}$, respectively, by using matrix $A$, while Eq. (6) relates $\mathbf{K}$ and $\mathbf{K}^{*}$ by using the inverse matrix of $A, A^{-1}$ :

$$
A=\left[\begin{array}{lll}
1 & 0 & a \\
0 & 1 & b \\
0 & 0 & c
\end{array}\right], \quad A^{-1}=\left[\begin{array}{ccc}
1 & 0 & -(a / c) \\
0 & 1 & -(b / c) \\
0 & 0 & 1 / c
\end{array}\right] .
$$

To obtain the physical representations of $a, b$, and $c$, we write $A=A_{3} A_{2} A_{1}$, where

$$
\begin{aligned}
& A_{1}=\left[\begin{array}{lll}
1 & 0 & a \\
0 & 1 & 0 \\
0 & 0 & 1
\end{array}\right], \\
& A_{2}=\left[\begin{array}{lll}
1 & 0 & 0 \\
0 & 1 & b \\
0 & 0 & 1
\end{array}\right], \\
& A_{3}=\left[\begin{array}{lll}
1 & 0 & 0 \\
0 & 1 & 0 \\
0 & 0 & c
\end{array}\right] .
\end{aligned}
$$



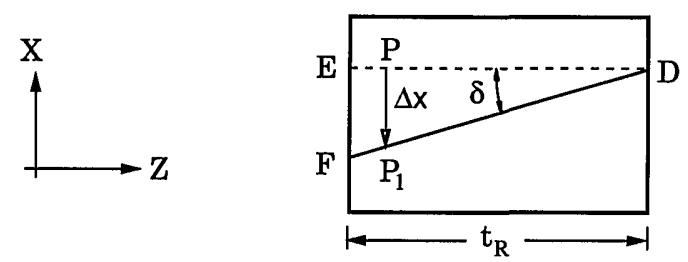

(a)

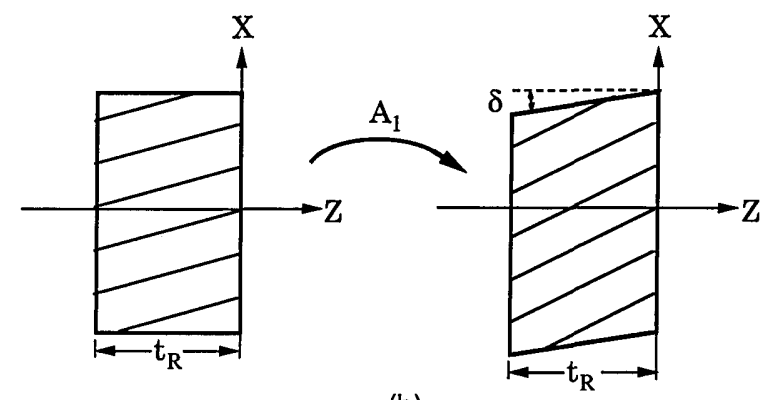

(b)

Fig. 4. (a) Action of the $A_{1}$ matrix on a point of the medium. (b) Grating before and after the transformation owing to the $A_{1}$ matrix.

For point $\mathrm{P}$ of the medium before processing, matrix $A_{1}$ transforms this point into another point, $\mathrm{P}_{1}$, so that $\Delta x=$ $a z, \Delta y=0$, and $\Delta z=0$, as we can see in Fig. 4(a). In this figure, segment $D E$ is transformed into segment $D F$; however, the thickness of the medium does not vary $(\Delta z=0)$. This shearing effect (a change in form but not in volume) is shown in Fig. 4(b). If $\delta$ is the angle between segments $D E$ and $D F$, then $\tan \delta=\Delta x / z=a z / z=a$.

From a physical point of view it is clear that the shear effect is due to the inclination itself of the Bragg planes, and therefore angle $\delta$ must be a function of angle $\phi$. This lateral motion can be explained by considering the lateral shearing forces that turn the Bragg planes or by considering the tendency of the medium to move laterally owing to the planes themselves. For bleached emulsions, Lamberts ${ }^{12}$ pointed out the existence of the lateral shrinkage forces of drying gelatin and that these shearing forces apparently become rather strong. Moreover, $\delta$ will be small (only a few degrees) owing to the elasticity of the material (in the emulsions, especially in gelatin bases). For Kodak $649 \mathrm{~F}$ plates, Kubota ${ }^{11}$ pointed out that it is possible artificially to obtain a lateral shift of as much as $\sim 3 \mu \mathrm{m}$ at the emulsion surface by soaking the plate in tepid water for $10 \mathrm{~min}$ and then letting it dry while applying various magnitudes of centrifugal force. Since the thickness of this emulsion was found to be $\sim 15 \mu \mathrm{m}$, a lateral shift of $<3 \mu \mathrm{m}$ implies that $\delta<11^{\circ}$. Applying a centrifugal force to the emulsion, he obtained lateral shifts at the photographic emulsion surface of as much as $\sim 0.3 \mu \mathrm{m}$ $\left(\delta \sim 1.1^{\circ}\right)$ and $1.0 \mu \mathrm{m}\left(\delta \sim 3.8^{\circ}\right)$ measured on 4 in. $\times 5$ in. $(\sim 10 \mathrm{~cm} \times \sim 13 \mathrm{~cm})$ Kodak 649 F plates.

For matrix $A_{2}, b$ corresponds to the tangent of a shear angle in the $Y$ direction, $\varphi$, with $\tan \varphi=\Delta y / z=b z / z=b$.

The $A_{3}$ matrix transforms point $\mathrm{P}$ into $\mathrm{P}_{1}$, as we can see from Fig. 5(a). The thickness of the medium changes from $t_{R}$ to $t_{C}$ [Fig. 5(b)]. It is easy to see that $t_{C}=c t_{R}$ and $T=c=t_{C} / t_{R}$. Figure 6 shows the total action of the $A$ matrix on the grating.
Taking into account the physical representation of the parameters $a, b$, and $c$, we can write the $A$ matrix as

$$
A=\left[\begin{array}{ccc}
1 & 0 & \tan \delta \\
0 & 1 & \tan \varphi \\
0 & 0 & T
\end{array}\right]
$$

When the recorded grating satisfies the condition $d_{y}=0$, then it must be that $\varphi=0$ and $b=0$; and if there is no shear effect, $\delta=\varphi=0$, and thus $K_{x} *=K_{x}$ and $K_{z}{ }^{*}=K_{z} / T$, as we can see in the literature, ${ }^{2,13} K_{z}{ }^{*}$ being a function of the quotient of the real thicknesses $t_{C}$ and $t_{R}$.

\section{EFFECTIVE THICKNESS}

In Section 3 we found the relationship that exists between a holographic grating before and after processing by using $T, \delta$, and $\varphi$. While the value of $T$ can be determined through experimentation by measuring the physical thicknesses of the medium before and after processing, the direct measurement of $\delta$ is obviously quite complicated.

Therefore we now consider a grating recorded with collimated beams with wave vectors with the $Y$ components null $(\varphi=0)$. To relate the shear angle $\delta$ to the parameters

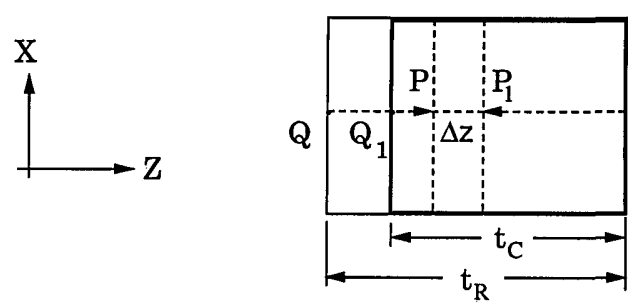

(a)

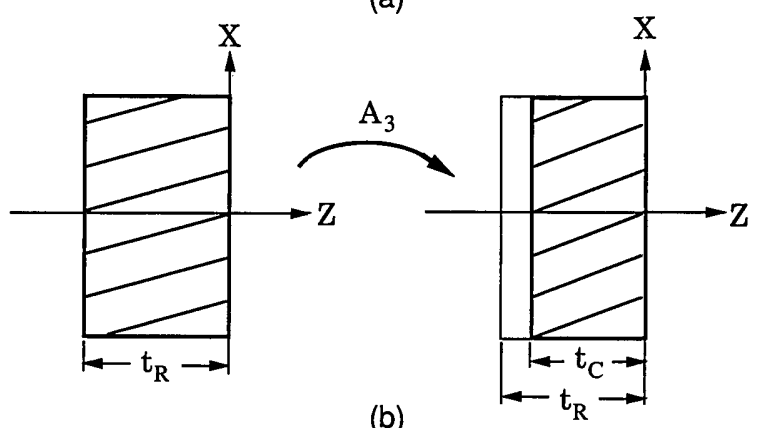

Fig. 5. (a) Action of the $A_{3}$ matrix on a point of the medium. (b) Grating before and after the transformation owing to the $A_{3}$ matrix.

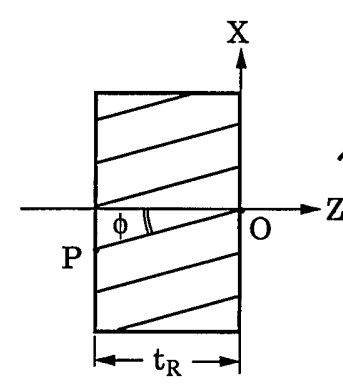

(a)

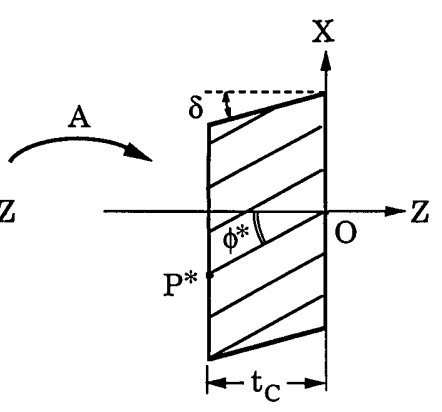

(b)
Fig. 6. Characteristic parameters of a holographic grating (a) before and (b) after the action of the $A$ matrix. 


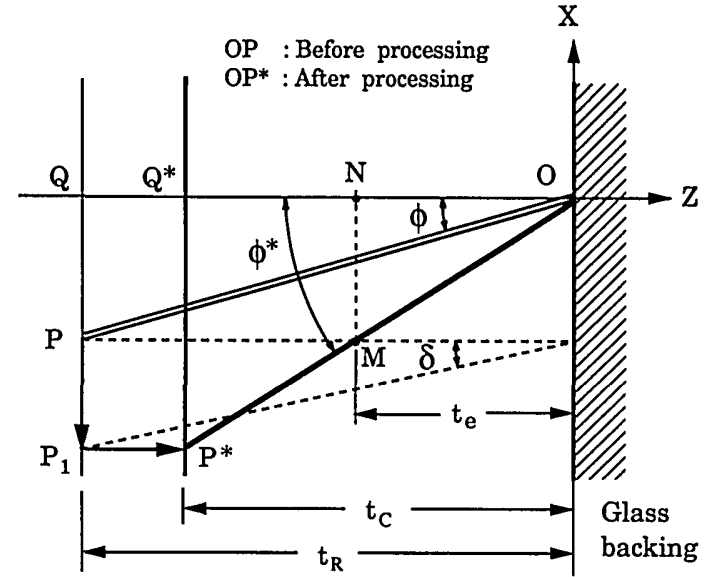

Fig. 7. Scheme to obtain the relationship between the grating parameters before and after processing.

characteristic of the grating, we refer to Fig. 7, which shows the change in a Bragg plane $O P$ that is due to processing when affected by the linear transformation given in Eq. (4). The relationship that exists between the coordinates of the points on the grating before and after processing can be expressed in the following manner:

$$
x^{*}=x+z \tan \delta, \quad z^{*}=T z .
$$

Similarly, the relationship between the grating vectors $\mathbf{K}$ and $\mathbf{K}^{*}$ is

$$
K_{x}^{*}=K_{x}, \quad K_{z}^{*}=\left(-K_{x} \tan \delta+K_{z}\right) / T .
$$

From Fig. 7 we can write

$$
\tan \delta=T \tan \phi^{*}-\tan \phi .
$$

By using the relation $\tan \phi=-K_{z} / K_{x}$ and Eq. (14), we can express Eq. (13) as

$$
K_{z}^{*}=\left(\tan \phi^{*} / \tan \phi\right) K_{z} .
$$

It is easy to see that $\tan \phi^{*} / \tan \phi=t_{R} / t_{e}$, where $t_{e}=O N$. If we say that $T_{e}=t_{e} / t_{R}$, then $\tan \phi^{*} / \tan \phi=1 / T_{e}$, and thus Eq. (15) becomes

$$
K_{z}^{*}=K_{z} / T_{e} .
$$

This equation is similar to the expression $K_{z}{ }^{*}=K_{z} / T,{ }^{2,13}$ except that $T_{e}$ is not the quotient of real thicknesses before and after processing. We call the $t_{e}$ parameter the effective thickness of the holographic grating.

The effective thickness plays a fundamental role in thick holographic gratings because it is directly related to the reconstruction angle that complies with Bragg's law. If this angle is $\alpha_{C}$, the following equation can be easily obtained for transmission gratings ${ }^{14}$ :

$$
\sin \alpha_{C}=\frac{1}{2}\left(\frac{N}{T_{e}}+\mu\right) \sin \alpha_{R}+\frac{1}{2}\left(\frac{N}{T_{e}}-\mu\right) \sin \alpha_{O},
$$

and it is possible to determine the $T_{e} / N$ quotient through experimentation by using

$$
\frac{T_{e}}{N}=\frac{\sin \alpha_{R}+\sin \alpha_{O}}{2 \sin \alpha_{C}+\mu\left(\sin \alpha_{O}-\sin \alpha_{R}\right)},
$$

where $N=n_{C} / n_{R}$ and $\mu=\lambda_{C} / \lambda_{R}$. The measurement of the recording angles and the reconstruction angle in compliance with Bragg's law gives not the actual thickness but rather the effective thickness. For reflection gratings, ${ }^{14}$ $T_{e}=\mu / N$. Nevertheless, in cases of reflection, good experimental results for $T$ can be obtained with the $T=$ $\mu / N$ equation.

Taking into account Eqs. (12)-(16), we express the parameters $\delta$ and $T_{e}$ as follows:

$$
\begin{aligned}
\tan \delta & =\frac{T-T_{e}}{T_{e}} \tan \phi, \\
T_{e} & =\frac{\tan \phi}{\tan \delta+\tan \phi} T .
\end{aligned}
$$

Equations (19) and (20) produce the following conclusions:

- The greater the difference between the angles $\delta$ and $\phi$, the more similar the values of $T$ and $T_{e}$.

- When $\phi$ is small, the difference between $T$ and $T_{e}$ is greater.

- When $\phi$ is $\sim 90^{\circ}$ (reflection grating), the $T$ and $T_{e}$ parameters coincide, so that, for reflection gratings, $T_{e} \approx T$.

- When $\phi \approx \delta$, then $T_{e} \approx 0.5 T$.

- In symmetric gratings $\left(\phi=0^{\circ}\right), \delta$ will equal $0^{\circ}$.

As a numerical example we can use a grating with $\phi=10^{\circ}, T=0.99$, and $T_{e}=0.80$. For these values $\delta=$ $2.4^{\circ}$ and $\phi^{*}=12.4^{\circ}$. A grating that is $6 \mu \mathrm{m}$ thick, $\delta=2.4^{\circ}$, produces a maximum lateral displacement $\left(\sim \delta \tan t_{R}\right)$ of $0.25 \mu \mathrm{m}$. This value is negligible compared with the

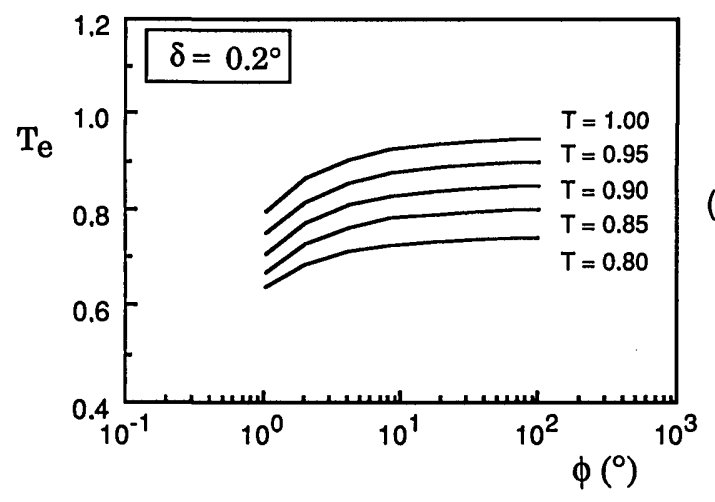

(a)

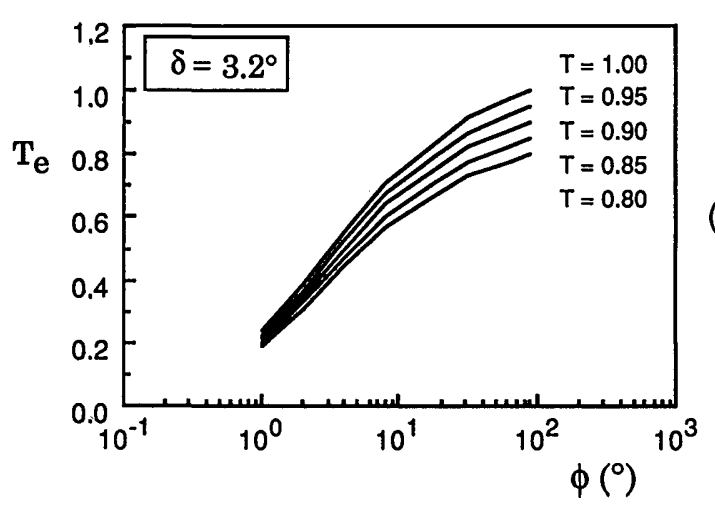

(b)

Fig. 8. $T_{e}$ as a function of the slant angle $\phi$ for different values of parameter $T$ and two fixed values of the shear angle: (a) $\delta=$ $0.2^{\circ}$; (b) $\delta=3.2^{\circ}$. 


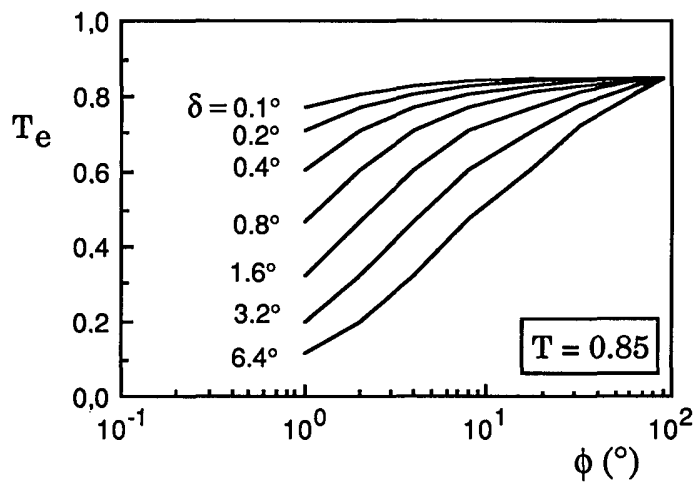

Fig. 9. $T_{e}$ as a function of the slant angle $\phi$ for $T=0.85$ and different values of the shear angle $\delta$.

longitudinal size of a grating that measures a few centimeters. Nevertheless, we will see how this small shearing effect $(\sim 0.0125 \%$ in a $2-\mathrm{cm}$ grating) can become of major importance in the reconstruction angle for maximum diffraction efficiency.

\section{SOME THEORETICAL RESULTS}

For every real holographic grating there is a corresponding effective holographic grating that has a $t_{e}$ thickness and that determines the direction of the diffracted beam. The effective thickness will not remain constant for the same type of recording material and the same value of the inclination angle of the fringes, because the deformations of the medium, and, consequently, $T$ and $\delta$, are a function of the recording material used, the type of processing done, the exposure, the relation of beams and spatial frequency. All these factors condition the elastic characteristics of the recording material.

In Fig. 8 we show some theoretical conclusions of the model. The effective thickness is represented as a function of the slant angle $\phi$, using Eq. (20), at fixed shear angles $\delta=0.2^{\circ}$ and $3.2^{\circ}$. Figure 9 shows $T_{e}$ as a function of the slant angle $\phi$ at a fixed real thickness $T=0.85$ and different shear angles. In all the $T_{e}$ curves shown, the $T_{e}$ values approximate those of $T$ the closer the $\phi$ angle gets to $90^{\circ}$, which is to say as a situation of reflection is approached. This is an important fact because it indicates that the data of reconstruction wavelength variation in reflection holographic gratings, for peak diffraction efficiency, are directly related to the real or physical thicknesses of the material after processing (for reflection gratings we can write $\left.T_{e} \cong T=\mu / N\right)$. From Fig. 9 we can see that, with low $\phi$ values, there are great differences in the values of $T_{e}$; while, when $\phi$ is closer to $90^{\circ}$, all the curves tend to have a value of $T=0.85$, independently of the value of $\delta$. These figures show that shear-type deformations, despite being negligible in terms of relative displacement, can indeed be significant in terms of angles, as long as the slant angle is not large (as is the case in transmission holograms).

\section{EXPERIMENTAL RESULTS}

To test the model, we formed transmission holographic gratings with two collimated beams of equal intensities from a He-Ne laser $\left(\lambda_{R}=633 \mathrm{~nm}\right)$ in Agfa Gevaert 8E75 HD photographic emulsions in the configuration illustrated in Fig. 10(a). The gratings were recorded with different exposures and with interbeam angles $\left|\alpha_{R}\right|+\left|\alpha_{O}\right|$ (in air) of $50^{\circ}, 40^{\circ}$, and $30^{\circ}$. For each recording geometry eight gratings were made, each with increased exposure values. A total of 240 transmission holographic gratings were made, half to be processed with rehalogenating bleach and the other half to be processed with reversal bleach. In all cases $\phi<14^{\circ}$, because it is precisely with small values of $\phi$ that the model proposed by Vilkomerson and Bostwick ${ }^{1}$ did not produce satisfactory results.

(a)

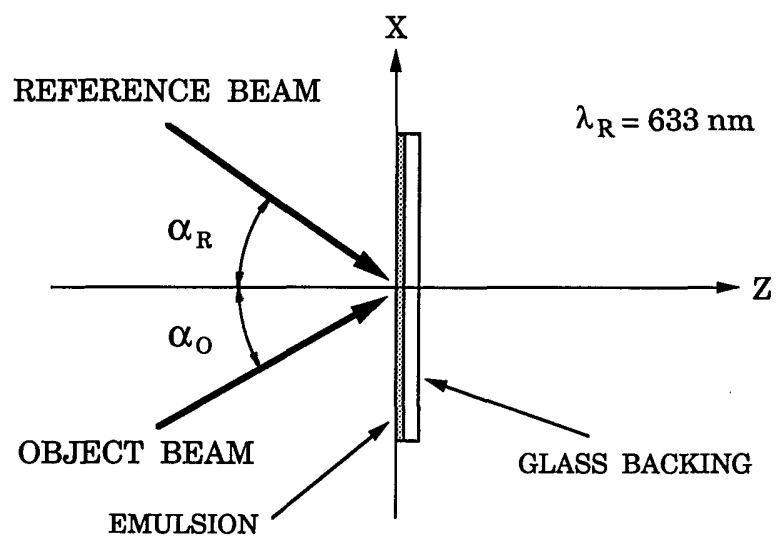

(b)

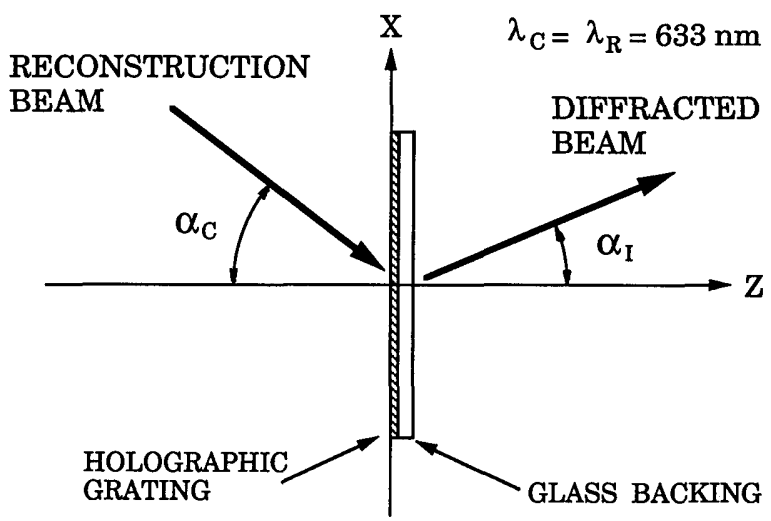

Fig. 10. Arrangement for (a) recording and (b) reconstructing holographic gratings.

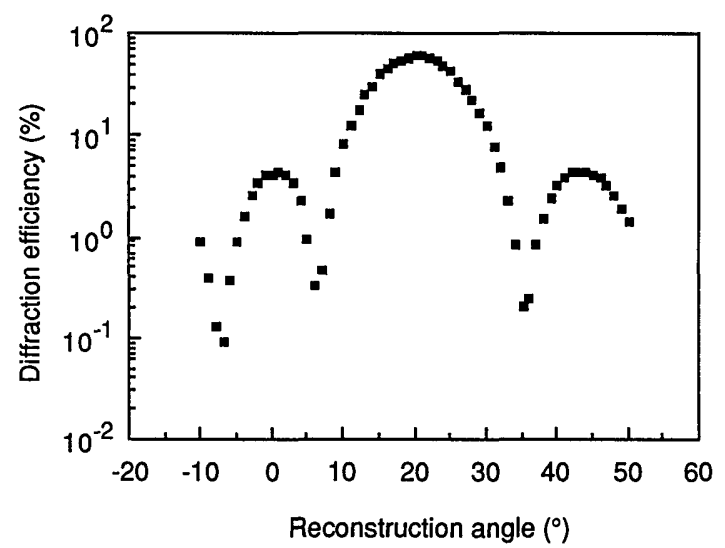

Fig. 11. Typical experimental results of the angular sensitivity measurements of holographic gratings. 


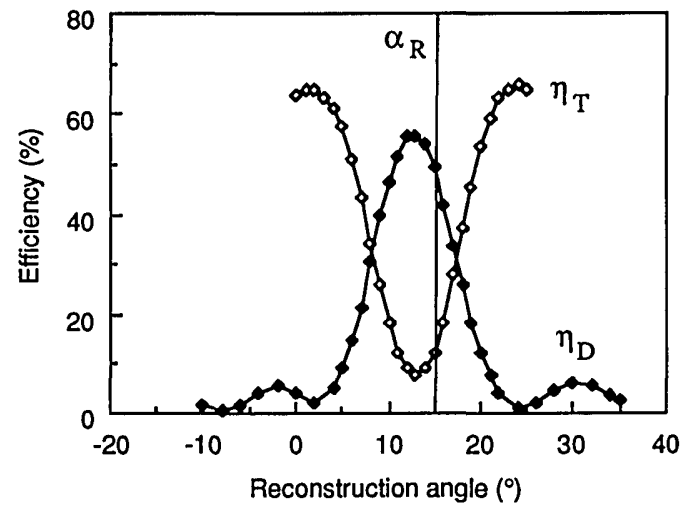

(a)

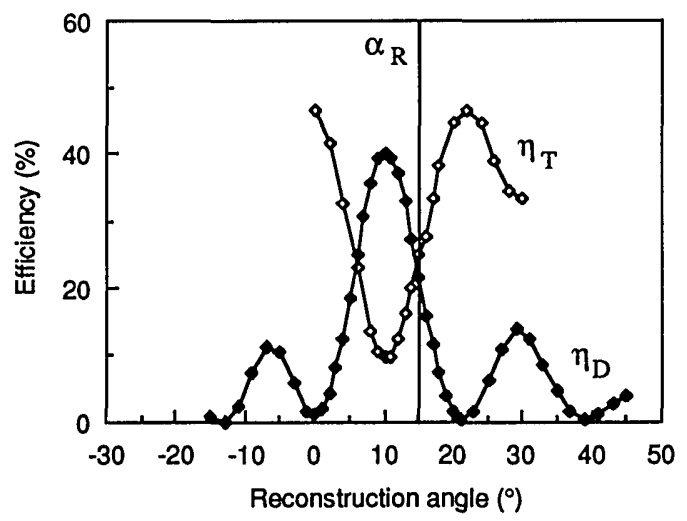

(b)

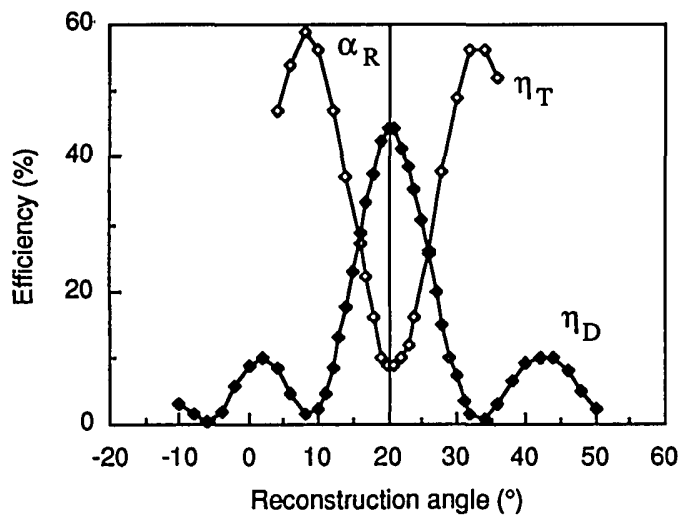

(c)

Fig. 12. Diffraction efficiency $\left(\eta_{D}\right)$ and transmission efficiency $\left(\eta_{T}\right)$ as functions of the reconstruction angle for the gratings processed with rehalogenating bleaching: (a) $E=324 \mu \mathrm{J} / \mathrm{cm}^{2}$, $\alpha_{R}=15.0^{\circ} \pm 0.1^{\circ}, \alpha_{O}=-35.0^{\circ} \pm 0.1^{\circ}, \phi=5.8^{\circ} \pm 0.1^{\circ}, f=$ 1315 lines $/ \mathrm{mm}$, Bragg angle $=12.9^{\circ} \pm 0.1^{\circ}, T_{e} / N=0.82 \pm 0.01 ;$ (b) $E=574 \mu \mathrm{J} / \mathrm{cm}^{2}, \alpha_{R}=15.0^{\circ} \pm 0.1^{\circ}, \alpha_{O}=-25.0^{\circ} \pm 0.1^{\circ}, \phi=$ $3.0^{\circ} \pm 0.1^{\circ}, f=1076$ lines $/ \mathrm{mm}$, Bragg angle $=10.2^{\circ} \pm 0.1^{\circ}, T_{e} / N=$ $0.50 \pm 0.02 ;$ (c) $E=324 \mu \mathrm{J} / \mathrm{cm}^{2}, \alpha_{R}=20.0^{\circ} \pm 0.1^{\circ}$, $\alpha_{O}=-20.0^{\circ} \pm 0.1^{\circ}, \phi=0.0^{\circ} \pm 0.1^{\circ}, f=1081$ lines $/ \mathrm{mm}$, Bragg angle $=20.0^{\circ} \pm 0.1^{\circ}$.

The exposed plates were developed in AAC developer with ascorbic acid and sodium carbonate. The developed plates were then rinsed briefly and bleached without a fixation step. ${ }^{15}$ Two types of bleach bath were used in these experiments. One was R-9, a reversal bleach. ${ }^{16}$ The other was $\mathrm{R}-10$, a rehalogenating bleach bath. ${ }^{17}$ R-9 bleach acts harshly owing to its solvent action, and this produces an important thickness variation. ${ }^{3}$ On the other hand, the resulting emulsion thickness change introduced with the rehalogenating bath is very small ${ }^{3,18}$ $(<0.05 \mu \mathrm{m})$ in the nominally 6 - $\mu \mathrm{m}$-thick film, ${ }^{8}$ and this implies that $T>0.992$.
The processed gratings were reconstructed with light from the same He-Ne laser $\left(\lambda_{C}=\lambda_{R}=633 \mathrm{~nm}\right)$, and the reconstruction angle that would provide maximum efficiency was obtained. To find this angle, we determined the angular response of the system by measuring not only the diffraction efficiency but also the efficiency of the zero-order transmitted beam. The reconstruction of the gratings was carried out according to the geometry of Fig. 10(b). The measurements of the angles were taken by using a rotation stage with a precision of $0.02^{\circ}$, also used in recording, which ensured sufficient sensitivity.

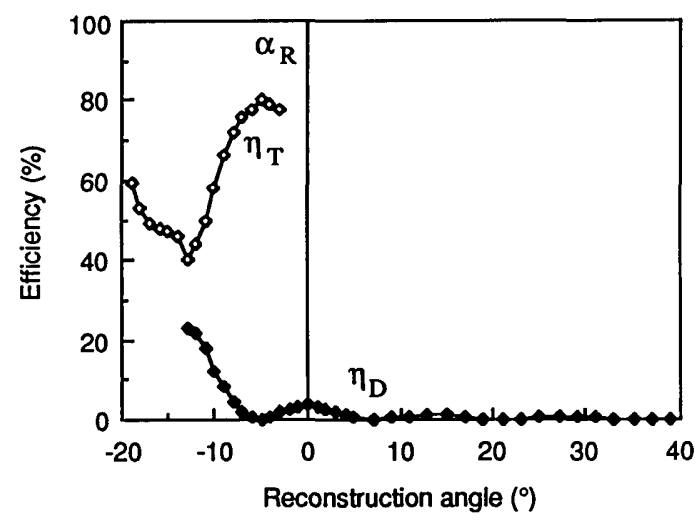

(a)

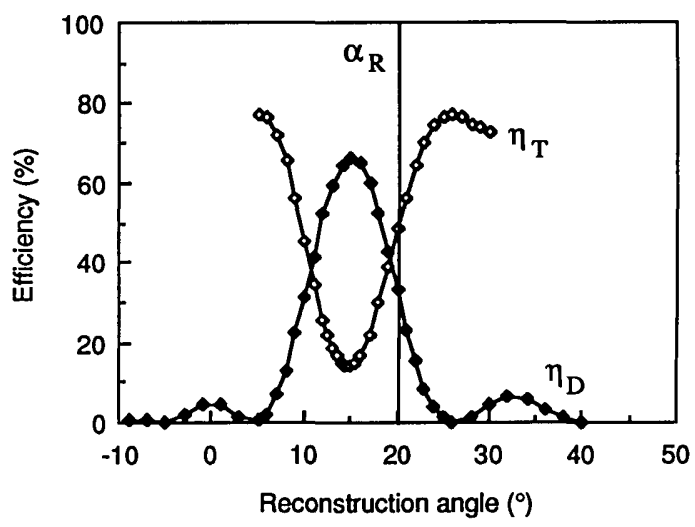

(b)

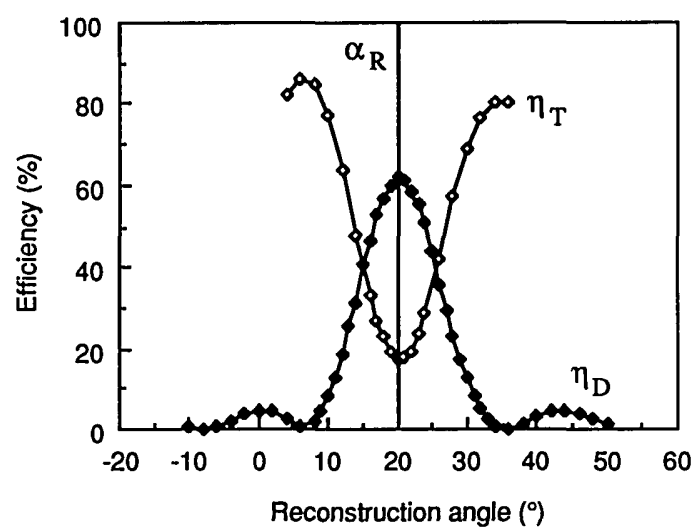

Fig. 13. Diffraction efficiency $\left(\eta_{D}\right)$ and transmission efficiency $\left(\eta_{T}\right)$ as functions of the reconstruction angle for the gratings processed with reversal bleaching: (a) $E=144 \mu \mathrm{J} / \mathrm{cm}^{2}, \alpha_{R}=$ $0.0^{\circ} \pm 0.1^{\circ}, \alpha_{O}=-50.0^{\circ} \pm 0.1^{\circ}, \phi=14.1^{\circ} \pm 0.1^{\circ}, f=1210$ lines/ $\mathrm{mm}$, Bragg angle $=-13.5^{\circ} \pm 0.1^{\circ}, T_{e} / N=0.621 \pm 0.005 ;$ (b) $E=187 \mu \mathrm{J} / \mathrm{cm}^{2}, \alpha_{R}=20.0^{\circ} \pm 0.1^{\circ}, \alpha_{O}=-30.0^{\circ} \pm 0.1^{\circ}$, $\phi=2.9^{\circ} \pm 0.1^{\circ}, f=1330$ lines $/ \mathrm{mm}$, Bragg angle $=14.5^{\circ} \pm 0.1^{\circ}$, $T_{e} / N=0.46 \pm 0.02 ;$ (c) $E=312 \mu \mathrm{J} / \mathrm{cm}^{2}, \alpha_{R}=20.0^{\circ} \pm 0.1^{\circ}$, $\alpha_{O}=-20.0^{\circ} \pm 0.1^{\circ}, \phi=0.0^{\circ} \pm 0.1^{\circ}, f=1081$ lines $/ \mathrm{mm}$, Bragg angle $=20.0^{\circ} \pm 0.1^{\circ}$. 

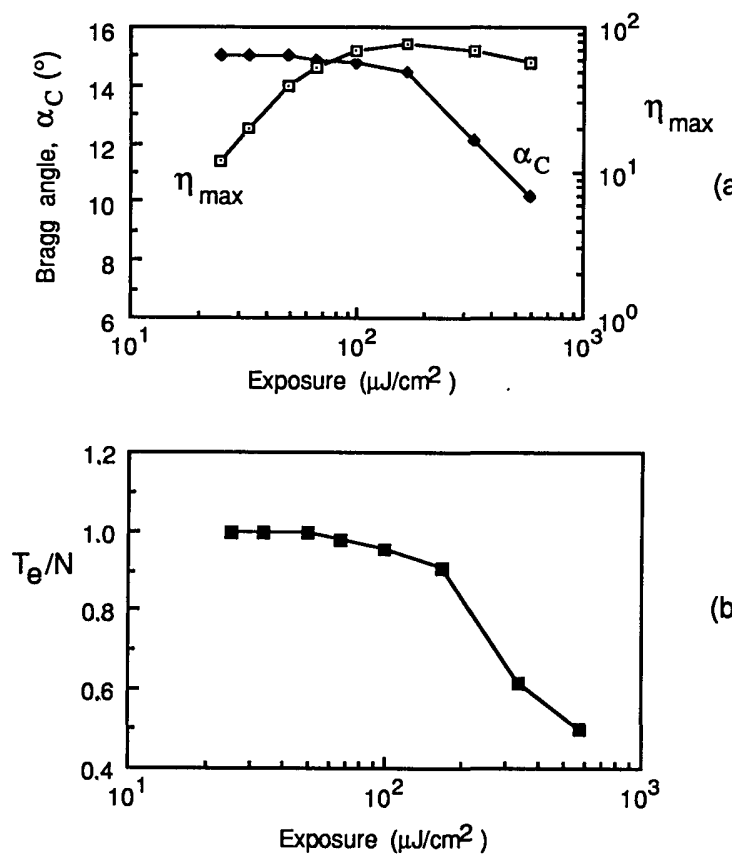

(b)

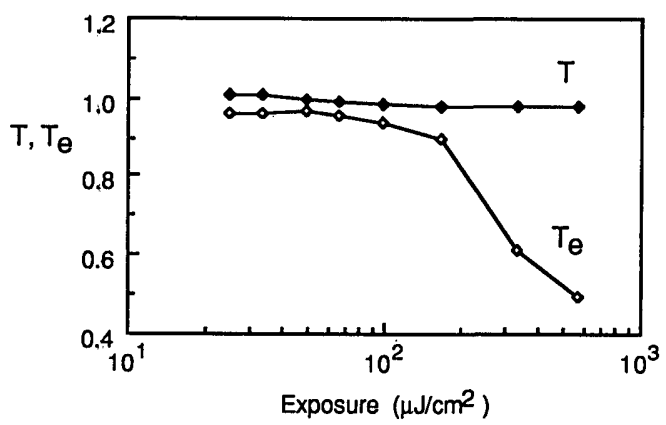

(c)

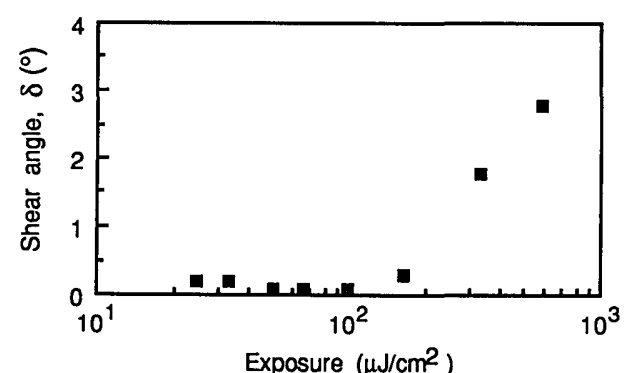

(d)

Fig. 14. Experimental results as a function of exposure of (a) the Bragg angle and the maximum diffraction efficiency, (b) $T_{e} / N$, (c) $T_{e}$ and $T$, and (d) the shear angle, $\delta$. The gratings were processed with rehalogenating bleaching. $\alpha_{R}=15.0^{\circ} \pm 0.1^{\circ}, \alpha_{O}=$ $-25.0^{\circ} \pm 0.1^{\circ}, \phi=3.0^{\circ} \pm 0.1^{\circ}, f=1076 \mathrm{lines} / \mathrm{mm}$.

We took $0.1^{\circ}$ as the absolute error of the angles because other possible sources of error such as the repositioning of the plates exist.

To obtain the $T_{e} / N$ quotient, we have shown the efficiencies of the beams transmitted and diffracted as a function of the reconstruction angle for all the recorded gratings. Likewise, we have shown the diffraction efficiency as a function of the reconstruction angle, on a logarithmic scale, to analyze the possible sidelobe asymmetries around the Bragg angle related to the bending of Bragg planes. ${ }^{11}$ In every case these curves show no appreciable deformation, providing clear support for the validity of hypothesis 1 of the model. In Fig. 11 we see a typical experimental result obtained from the angular sensitivity measurement of the transmission holographic grating. This figure does not show sidelobe asymmetry.

We determined the value of the Bragg angle by adjusting the part of the curve of the diffraction efficiency as a function of the reconstruction angle, at its approximate maximum value, by using polynomic functions. If this angle is known, the $T_{e} / N$ quotient can be found by using Eq. (18) with $\mu=1$. However, to obtain $T_{e}$ it is necessary to know $N$ as a function of exposure. To find $N$ we used the method proposed by Lamberts ${ }^{12}$ of measuring the refractive indices of photographic emulsions, considering that the average refractive index of the unprocessed emul-

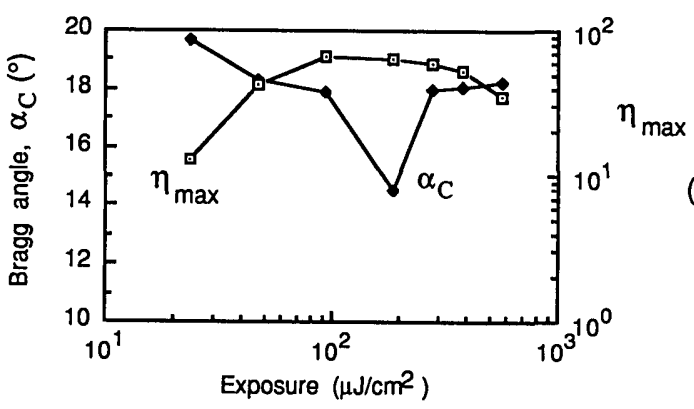

(a)

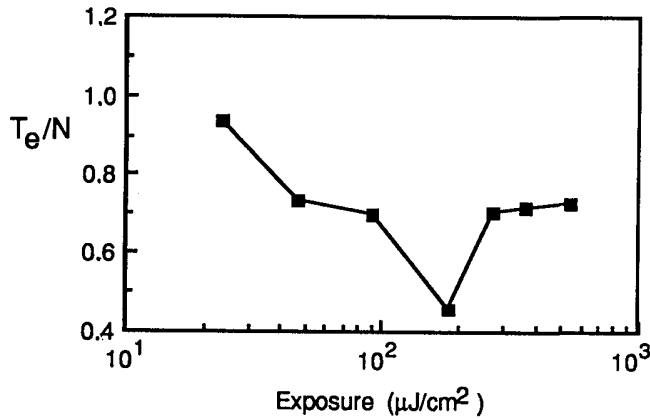

(b)

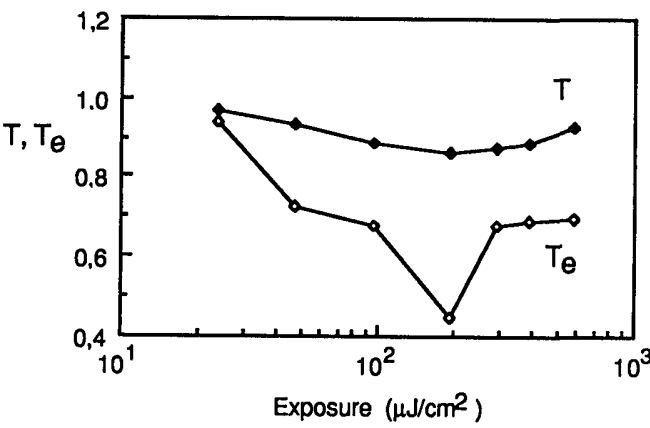

(c)

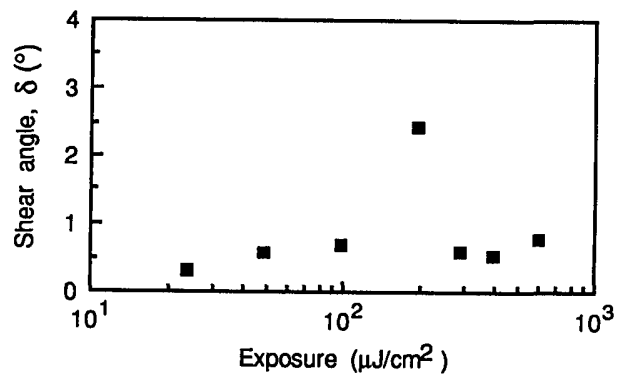

Fig. 15. Experimental results as a function of exposure of (a) the Bragg angle and the maximum diffraction efficiency, (b) $T_{e} / N$, (c) $T_{e}$ and $T$, and (d) the shear angle, $\delta$. The gratings were processed with reversal bleaching. $\alpha_{R}=20.0^{\circ} \pm 0.1^{\circ}, \alpha_{O}=$ $-30.0^{\circ} \pm 0.1^{\circ}, \phi=2.9^{\circ} \pm 0.1^{\circ}, f=1330$ lines $/ \mathrm{mm}$. 
sion is $n_{R}=1.64$ at $633 \mathrm{~nm} .^{19}$ We obtained values for the parameter $N$ of between 0.95 and 1.00 with an error of 0.01 .

On the other hand, to compare the values of $T$ and $T_{e}$ it is necessary to know $T$ as a function of exposure. Taking into account that for reflection gratings $T_{e} \approx T$, we record reflection gratings in Agfa Gevaert 8E75 HD emulsion, using the same wavelength and processing as used in the transmission gratings and measuring the reconstruction wavelength that complies with Bragg's law. ${ }^{13}$ When this wavelength and the values of $N$ measured above are known, it is possible to obtain $T$ as a function of exposure by using the equation $T=\mu / N$. From these experiments we conclude that, with the reversal bleach, emulsion shrinkage increases with exposure, while with the rehalogenating bleach the change in emulsion thickness with exposure is minimal. ${ }^{3}$

Figures 12 and 13 show some examples of the measurements of the diffraction efficiency $\left(\eta_{D}\right)$ and the transmission efficiency $\left(\eta_{T}\right)$ together with the values of the Bragg angle and the $T_{e} / N$ quotient. In these figures the vertical line is placed on the reconstruction angle, which is the same as the reference beam angle. The gratings that were processed with rehalogenating bleach [Figs. 12(a) and 12(b)] have values of $T_{e} / N$ of $0.82 \pm 0.01$ and $0.50 \pm$ 0.02 , respectively, and those processed with a reversal bleach [Figs. 13(a) and 13(b)] have values of $0.621 \pm 0.005$ and $0.46 \pm 0.02$, respectively. In no case can these values correspond to real thickness quotients.

For symmetrical cases [Figs. 12(c) and 13(c)], that is to say, when $\alpha_{R}=-\alpha_{O}$, the Bragg angle virtually coincides with the reconstruction angle, as indicated in Section 5 . Except in these symmetrical cases, in all the cases that we analyzed, a displacement of the reconstruction angle for maximum diffraction efficiency with respect to the angle of the reference beam is observed. In the experiments that were done, some interesting situations were observed, such as the one shown in Fig. 13(a). For this grating, maximum efficiency should be $65 \%$, yet only $20 \%$ was achieved. In this case, the rotation of the Bragg planes was so great that for the Bragg angle the diffracted beam eventually ended up tangent to the plate.

Figures 14 and 15 are two examples of sets of experimental graphs in which we represented the following items as a function of exposure: (a) the maximum diffraction efficiency $\left(\eta_{\max }\right)$ and the Bragg angles; (b) the values of $T_{e} / N$ obtained by applying Eq. (18); (c) the comparison of $T_{e}$ and $T$; and (d) the $\delta$ angles, calculated with Eq. (19). In all the cases that we analyzed, the relative errors of $T_{c} / N$ and $T_{e}$ are less than $5 \%$, while the relative error of $\delta$ is less than $10 \%$.

By analyzing all the graphs that we obtained, which were similar to those in Figs. 14 and 15, we can draw some conclusions about these bleaches:

1. A comparison of the $T$ and the $T_{e}$ values indicates that $T_{e}<T$. For rehalogenating bleach $T \sim 1$ and $0.4<$ $T_{e}<1$. For reversal bleach $T \sim 0.9$ and $0.5<T_{e}<1$. We can see that lower values of $T_{e}$ are obtained with rehalogenating bleach when the $T$ values are higher. We believe that with this bleach some intense shearing effects appear and that they cause significant lateral displacement with $\delta$ values between $0^{\circ}$ and $5^{\circ}$.
2. The values of $\delta$ are low $\left(<8^{\circ}\right)$, and in most cases they do not even reach $3^{\circ}$, which gives rise to small but significant lateral displacement (see Section 5).

3. In all cases the lowest values of $T_{e}$ correspond to the geometries that are the most symmetric (lower value of the $\phi$ angle), just as was predicted theoretically (see Figs. 8 and 9).

4. To suppose that $T_{e}$ is the true thickness variation, $T$, would lead us to the physically impossible result that the photochemical processes used could produce shrinkage levels of more than $50 \%$.

5. $T_{e}$ is a function of recording geometry, exposure, and processing. This is one of the ways in which the EHGM can be used in the analysis of chemical processing, because $T_{e}$ gives us information about processing. For example, in rehalogenating bleach, thickness variation is quite small but $T_{e}$ can vary greatly. Therefore, with this bleach, material can transfer from one zone to another ${ }^{20}$ with no change in volume but with an important shear effect.

\section{CONCLUSIONS}

In this paper we introduced a new model for analyzing holographic gratings (the EHGM) that is more comprehensive because it takes into account deformations in the recording material that were not previously contemplated in the literature. These deformations can play a fundamental role in the reconstruction geometry of a holographic grating for maximum diffraction efficiency. With this model it is possible to explain the anomalous values of shrinkage, keeping in mind that these values correspond to a shrinkage effect (described by $T$ ) and a shear effect (described by $\delta$ ). This allows for a new parameter in the model, the angle $\delta$, which was not previously dealt with in the literature. Taking this into account, one can characterize the deformation of the recording medium that is due to processing by using two parameters, $T$ and $\delta$. Another parameter introduced in the model is the effective thickness, characteristic of recording material deformations that are due to processing, which allows us to compare the components of the grating vector before and after processing. Since reconstruction geometry for maximum diffraction efficiency depends on the grating vector, this effective thickness conditions the reconstruction stage of the holographic grating.

Parameters $T, T_{e}$, and $\delta$ are related through Eq. (20). Experimentally it is possible to find the values of $T$ (for example, by using reflection gratings) and $T_{e}$ (by measuring the Bragg angle before processing). It is important to point out that, when the slant angle $\phi$ is high, the values of the $T$ and the $T_{e}$ parameters practically coincide. Using Eq. (20), we can estimate the shear angle $\delta$. As we pointed out in Section 3, the values obtained for this angle are small. On the other hand, the model justifies the experimental results that were obtained in the analysis of more than 240 transmission holographic gratings submitted to both rehalogenating and reversal bleaches, some of which have been cited as examples in this paper; furthermore, by using the model we can obtain information about processing if we know the value of $T_{e}$.

In the model that we presented, we have studied a homogeneous deformation of the material, but the model can 
be generalized and applied to cases in which the Bragg planes are curved. ${ }^{11,21}$ Such a situation would imply that $K_{z}{ }^{*}$ is a function of $z$ and that the coefficients of transformation matrix $A$ are not constants.

Finally, it is possible to apply the EHGM to holographic lenses ${ }^{14}$ by using an approximation of the local grating that produces a set of relationships between the coordinates of the recording and the reconstruction sources when spherical wave fronts are used and Bragg's law is complied with in the reconstruction stage. ${ }^{22}$ In these expressions the $T_{e}, N$, and $\mu$ parameters play similar roles. In such a case it is possible to find geometries in which the combinations of these three parameters give way to situations in which the aberrations that are due to changes in geometry and wavelength are minimized and, at the same time, high diffraction efficiencies are achieved. ${ }^{22}$ This introduces a new area in the field of HOE design in which the recording material plays an essential role.

\section{ACKNOWLEDGMENTS}

The authors gratefully acknowledge the technical assistance provided by the Holography Center of Alicante. The authors express their gratitude to the topical editor, H. J. Caulfield, for the attention he gave this paper. Thanks are also due to the reviewers for their useful suggestions and for their comments, which significantly improved the original manuscript.

A. Beléndez is also with the Departamento de Ingeniería de Sistemas y Communicaciones of the University of Alicante, Alicante, Spain.

\section{REFERENCES}

1. D. H. R. Vilkomerson and D. Bostwick, "Some effects of emulsion shrinkage on a hologram's image space," Appl. Opt. 6, 1270-1272 (1967).

2. I. A. Mikhailov, "A geometrical analysis of thick holograms," Opt. Spectrosc. (USSR) 58, 374-376 (1985).

3. P. Hariharan and C. M. Chidley, "Bleached reflection holograms: a study of color shifts due to processing," Appl. Opt. 28, 422-424 (1989).

4. R. R. A. Syms and L. Solymar, "Experimental and theoretical evaluation of the efficiency of an off-axis volume holographic lens," Appl. Phys. B 32, 165-173 (1983).
5. A. Beléndez, I. Pascual, and A. Fimia, "Experimental results in thickness and index variations in the analysis of holographic aberrations," in Optics in Complex Systems, F. Lanzl, H. Preuss, and G. Weigelt, eds., Proc. Soc. Photo-Opt. Instrum. Eng. 1319, 305 (1990).

6. P. Hariharan, Optical Holography (Cambridge U. Press, Cambridge, 1984), Chap. 7, p. 92.

7. I. A. Mikhailov, "Characteristic properties of the recording and interference copying of thick transmission holograms," Opt. Spectrosc. (USSR) 64, 224-226 (1988).

8. R. K. Kostuk, "Factorial optimization of bleach constituents for silver halide holograms," Appl. Opt. 30, 1611-1616 (1991).

9. H. Kogelnik, "Coupled wave theory for thick hologram gratings," Bell. Syst. Tech. J. 48, 2909-2947 (1969).

10. T. Kubota, "Characteristics of thick hologram gratings recorded in absorptive mediums," Opt. Acta 25, 1035-1053 (1978).

11. T. Kubota, "The bending of interference fringes inside a hologram," Opt. Acta 26, 731-743 (1979).

12. R. L. Lamberts, "Characterization of a bleached photographic material," Appl. Opt. 11, 33-41 (1972).

13. Y. T. Huang, M. Kato, and R. K. Kostuk, "Methods for fabricating substrate-mode holographic optical elements," in Computer and Optically Formed Holographic Optics, I. Cindrich and S. H. Lee, eds., Proc. Soc. Photo-Opt. Instrum. Eng. 1211, 166-174 (1990).

14. A. Beléndez, "Influences of recording material on the characteristics of holographic optical elements," Ph.D. dissertation (Valencia University, Valencia, Spain, 1990).

15. J. Crespo, A. Fimia, and J. A. Quintana, "Fixation-free methods in bleached reflection holography," Appl. Opt. 25, 16421645 (1986).

16. R. L. Lamberts and C. N. Kurtz, "Reversal bleaching for low flare light in holograms," Appl. Opt. 10, 1342-1347 (1971).

17. L. Joly, "Grain growth during rehalogenating bleaching," J. Photogr. Sci. 31, 143-147 (1983).

18. R. K. Kostuk and G. T. Sincerbox, "Polarization sensitivity of noise gratings recorded in silver halide volume holograms," Appl. Opt. 27, 2993-2998 (1988).

19. R. K. Kostuk and J. W. Goodman, "Refractive index modulation mechanism in bleached silver halide holograms," Appl. Opt. 30, 369-371 (1991).

20. P. Hariharan, "Rehalogenating bleaches for photographic phase holograms. 3: Mechanism of material transfer," Appl. Opt. 29, 2983-2985 (1990).

21. P. Fiala, J. Ruzek, and T. Jerie, "Behavior and properties of real holographic recording materials," in Practical Holography II, T. H. Jeong, ed., Proc. Soc. Photo-Opt. Instrum. Eng. 747, 74-81 (1987).

22. A. Beléndez, I. Pascual, and A. Fimia, "Optimization of reconstruction geometry for maximum diffraction efficiency in HOE: the influence of recording material," in International Colloquium on Diffractive Optical Elements, J. Nowak, ed., Proc. Soc. Photo-Opt. Instrum. Eng. 1574, 77-83 (1991). 